\title{
Influência da Chuva na Eficácia do Herbicida 2,4-D no Controle DE Myriophyllum aquaticum ${ }^{1}$
}

\author{
Influence of Rain on Herbicide 2,4-D Efficacy in Controlling Myriophyllum aquaticum
}

SOUZA, G.S.F. ${ }^{2}$, PEREIRA, M.R.R. ${ }^{3}$, VITORINO, H.S. ${ }^{4}$, CAMPOS, C.F. ${ }^{5}$ e MARTINS, D. ${ }^{6}$

\begin{abstract}
RESUMO - O objetivo deste estudo foi avaliar a eficiência de controle de duas doses do herbicida 2,4-D pulverizado em plantas de $M$. aquaticum e submetido à simulação de chuva em diferentes periodos de tempo após sua aplicação. O delineamento experimental utilizado foi o inteiramente casualizado, com quatro repetições, disposto em um esquema fatorial $2 \times 8$ (duas doses de herbicida e oito intervalos de chuva). Utilizou-se o herbicida 2,4-D, na formulação comercial DMA 806, em duas doses: 670 e 1.340 g e.a. ha-1 ${ }^{-1}$ A simulação de uma chuva de $15 \mathrm{~mm}$, com duração de cinco minutos, foi realizada em oito intervalos de tempo após a pulverização do herbicida $(0,25 \mathrm{~h}, 0,5 \mathrm{~h}, 1 \mathrm{~h}, 2 \mathrm{~h}, 4 \mathrm{~h}, 8 \mathrm{~h}, 12 \mathrm{~h}$ e sem chuva). Foram realizadas avaliações visuais de controle aos $7,14,21$ e 28 dias após a aplicação dos tratamentos. As duas doses avaliadas do herbicida 2,4-D foram eficientes no controle das plantas de M. aquaticum, mesmo com ocorrência de chuva 15 minutos após sua aplicação.
\end{abstract}

Palavras-chave: eficiência, planta aquática, planta daninha, precipitação.

\begin{abstract}
The objective of this study was to evaluate the efficiency of two doses of the herbicide 2,4-D sprayed on Myriophyllum aquaticum plants and submitted to simulated rainfall during different periods of time after application. The experiment was arranged in a completely randomized design, with four replications, in a factorial $2 \times 8$ (two herbicide doses and eight rain intervals). Herbicide 2, 4-D was applied in the formulation DMA 806 in two doses, 670 and 1,340 g a.e. hat ${ }^{-1}$. Simulation of $15 \mathrm{~mm}$ rain for 5 minutes was performed in eight time intervals after herbicide spraying $(0.25 \mathrm{~h}, 0.5 \mathrm{~h}, 1 \mathrm{~h}, 2 \mathrm{~h}, 4 \mathrm{~h}, 8 \mathrm{~h}, 12 \mathrm{~h}$, and no rain). Visual evaluations of control were carried out at 7 , 14,21 , and 28 days after treatment application. The two doses of the herbicide 2, 4-D were efficient in controlling the plants of $\boldsymbol{M}$. aquaticum, even with the occurrence of rainfall 15 minutes after application.
\end{abstract}

Keywords: efficiency, aquatic plant, weed, rainfall.

\section{INTRODUÇÃO}

A intervenção antrópica estabelecida de maneira não planejada sobre os diversos ecossistemas aquáticos promove uma série de alterações nas características químicas, físicas e bióticas dos corpos hídricos, levando a modificações expressivas na comunidade biótica que os coloniza, incluindo a expansão de populações de espécies de plantas aquáticas
(Souza et al., 2011). Enquanto algumas dessas espécies apresentam-se enraizadas em corpos d'água com fortes correntezas, outras somente podem viver em águas paradas ou estagnadas (Martins et al., 2002).

Myriophyllum aquaticum é uma planta perene, herbácea, originária da América do Sul e de ocorrência natural no Brasil; popularmente conhecida como pinheirinhod'água, desenvolve-se totalmente de forma

Recebido para publicação em 12.12.2011 e aprovado em 12.3.2012.

2 Engo-Agro ${ }^{\circ}$, M.Sc., Doutorando, Faculdade de Ciências Agronômicas, Universidade Estadual Paulista "Júlio de Mesquita Filho", FCA/UNESP, Caixa Postal 237, 18610-307 Botucatu-SP, <guilhermesasso@fca.unesp.br>; ${ }^{3}$ Engenheira Florestal, Dra., Pós-Doutoranda em Agronomia, FCA/UNESP, ${ }^{4}$ Eng $^{\mathrm{o}}-\mathrm{Agr}^{\mathrm{o}}$., M.Sc., Doutorando em Agronomia, FCA/UNESP, ${ }^{5}$ Eng $^{\mathrm{O}}-\mathrm{Agr}^{\mathrm{o}}$., Mestrando em Agronomia, FCA/UNESP; ${ }^{6}$ Professor Livre Docente, Dep. de Produção Vegetal, FCA-UNESP.

Planta Daninha, Viçosa-MG, v. 30, n. 2, p. 263-267, 2012 
submersa e pode apresentar a porção terminal dos ramos sob superfície, sendo variável conforme o nível da água (Kissmann, 1997). O ambiente propício ao seu desenvolvimento é a água doce, parada ou com fraca movimentação, com elevado teor de nutrientes, especialmente nitrogênio, e temperaturas entre 8 e $30^{\circ} \mathrm{C}$ (Domingos et al., 2005).

O controle mecânico é um método, na maioria das situações, eficiente no controle de plantas aquáticas (MacDonald \& Langeland, 2001), exceto em situações especiais, como é o caso de $M$. aquaticum, que se reproduz por sementes, fragmentos ou pequenas estruturas das plantas (Reynolds, 1999). Com essa situação, o controle químico passa a ser necessário e mais eficaz para o gerenciamento de infestações dessa espécie (Wersal et al., 2010). Alguns estudos avaliaram diversos herbicidas para o controle de $M$. aquaticum, como os herbicidas de contato diquat e endothall, os quais apresentaram curto prazo de controle e necessitaram de repetidas aplicações (Moreira et al., 1999). Wersal \& Madsen (2007) relataram controles de 50 e $100 \%$ das plantas de pinheirinhod'água com aplicações foliares dos herbicidas imazamox e imazapyr, respectivamente. Hofstra et al. (2006), ao avaliarem os herbicidas sistêmicos glyphosate, 2,4-D, triclopyr e fluridone para o controle de plantas de $M$. aquaticum em condição de mesocosmo, observaram que apenas o triclopyr, quando aplicado em taxas maiores que 2,0 kg e.a. ha-1, proporcionou controle completo da planta aquática. Outros trabalhos têm comprovado a eficiência do controle de plantas de $M$. aquaticum com a aplicação do herbicida 2,4-D, como os realizados por MacDonald \& Langeland (2001), Negrisoli et al. (2003), Gray et al. (2007) e Wersal \& Madsen (2010).

No entanto, após atingir a superficie foliar, os herbicidas estão sujeitos a vários destinos, como secar e formar substância amorfa, cristalizar após a evaporação do solvente ou, ainda, ser lavado pela ocorrência de chuva após sua aplicação (Werlang et al., 2003). A consequência imediata disso é a menor absorção e menor eficiência do herbicida (Souza et al., 2011).

O período crítico entre aplicação do herbicida em pós-emergência e ocorrência de chuvas varia de acordo com o tipo de formulação, a dose empregada, a solubilidade do produto na água, as espécies de plantas daninhas, as condições de desenvolvimento destas e a quantidade de chuva (Anderson \& Arnold, 1984).

Assim, o objetivo deste trabalho foi avaliar a influência da ocorrência de chuvas em diferentes períodos de tempo após a aplicação do herbicida 2,4-D na sua eficácia de controle sobre plantas de $M$. aquaticum.

\section{MATERIAL E MÉTODOS}

As mudas de $M$. aquaticum foram obtidas em um canal de drenagem de várzea, localizado na Fazenda Edgardia, pertencente à UNESP, campus de Botucatu-SP, e padronizadas com $20 \mathrm{~cm}$ de comprimento. Quatro mudas foram transplantadas e conduzidas em vasos plásticos de $20 \times 20 \times 25 \mathrm{~cm}$, preenchidos com $2,5 \mathrm{~kg}$ de solo adubado e com a água mantida a $5 \mathrm{~cm}$ acima do nível do solo, os quais foram deixados a pleno sol. O delineamento experimental utilizado foi o inteiramente casualizado, com quatro repetições, disposto em um esquema fatorial $2 \times 8$ (duas doses do herbicida 2,4-D e oito intervalos de chuva). Os tratamentos químicos constaram da aplicação do herbicida 2,4-D, na formulação comercial DMA $806 \mathrm{BR}$, em duas doses: 670 e 1.340 g e.a. ha ${ }^{-1}$. A simulação de uma chuva de $15 \mathrm{~mm}$, com duração de cinco minutos, foi realizada em oito intervalos de tempo após a pulverização dos herbicidas $(0,25 \mathrm{~h}, 0,5 \mathrm{~h}, 1 \mathrm{~h}, 2 \mathrm{~h}, 4 \mathrm{~h}, 8 \mathrm{~h}, 12 \mathrm{~h}$ e uma testemunha sem simulação de chuva).

A simulação da chuva foi feita sob um sistema estacionário de aplicação de água regulado para aplicação da chuva pretendida; a aplicação do herbicida foi realizada através de um pulverizador costal, com pressão constante de $\mathrm{CO}_{2}$, equipado com uma barra de aplicação munida de duas pontas tipo jato plano Teejet XR 11002VS, distanciadas de $0,50 \mathrm{~m}$ entre si e com consumo de calda de $200 \mathrm{~L} \mathrm{ha}^{-1}$.

As plantas foram avaliadas visualmente aos 7, 14, 21 e 28 dias após a aplicação (DAA), por meio de uma escala percentual de notas, em que zero representava nenhum controle e 
$100 \%$ o controle total das plantas (SBCPD, 1995). Os parâmetros utilizados para o estabelecimento das notas visuais de controle foram: acúmulo de biomassa, inibição do crescimento, quantidade e uniformidade das injúrias e capacidade de rebrota das plantas.

Os resultados foram submetidos à análise de variância pelo teste $F$, sendo as médias dos tratamentos comparadas pelo teste de Tukey $(\mathrm{p}<0,05)$.

\section{RESULTADOS E DISCUSSÃO}

$\mathrm{Na}$ primeira avaliação visual, realizada aos 7 DAA (Tabela 1), observa-se que as duas doses do herbicida 2,4-D estudadas proporcionaram elevadas porcentagens de intoxicação às plantas de $M$. aquaticum, independentemente da ocorrência de chuva após sua aplicação, com médias de controle acima de $80 \%$, com exceção apenas do tratamento com aplicação da menor dose de herbicida (670 g e.a. ha-1). Registra-se que apenas quando da aplicação da dose menor de 2,4-D (670 g e.a. ha ${ }^{-1}$ ) houve efeito dos períodos de chuva, o qual foi reduzido somente quando da ocorrência de chuva 15 minutos após sua aplicação. Ressalta-se também que apenas notou-se efeito da dose aplicada de 2,4-D quando da ocorrência de chuva 15 minutos após sua aplicação, com controle superior na maior dose testada. Gray et al. (2007), ao avaliarem a eficácia do herbicida 2,4-D no controle de plantas de $M$. aquaticum, relataram porcentagens médias de controle de $72 \%$ aos 7 DAA para a aplicação de $1.000 \mu \mathrm{g}$ e.a. $\mathrm{L}^{-1}$.
Aos 14 DAA, verificou-se que as médias de controle elevaram-se em todos os tratamentos avaliados (Tabela 2). O tratamento com aplicação da maior dose de 2,4-D não foi influenciado pela ocorrência de chuva em qualquer intervalo de tempo estudado após sua aplicação, apresentando médias de controle acima de 97\%. MacDonald \& Langeland (2001) também observaram excelente controle de Myriophyllum spp. com uso do herbicida 2,4-D.

Já o tratamento com aplicação da menor dose do herbicida foi afetado somente pela ocorrência de chuvas após 15 minutos de sua aplicação; nos demais intervalos de tempo entre sua aplicação e a ocorrência de chuva, proporcionou um controle mínimo de $98 \%$ das plantas de $M$. aquaticum. Resultados excelentes de controle dessa planta também foram obtidos em estudo com aplicação de diferentes doses do herbicida 2,4-D (4,4 e 8,9 kg p.c. ha-1) em folhas emergentes de plantas jovens (Washington, 1998).

Na avaliação realizada aos 21 DAA, notase o mesmo comportamento apresentado pelos tratamentos nas avaliações anteriores. A ocorrência de chuva após 15 minutos da aplicação da menor dose de 2,4-D (670 g e.a. ha $\left.{ }^{-1}\right)$ reduziu sua eficiência de controle (Tabela 3). No entanto, aos 28 DAA essa redução de eficácia do 2,4-D proporcionada por uma chuva ocorrida aos 15 minutos após a sua aplicação não influenciou mais a eficiência de controle das plantas de $M$. aquaticum pelo herbicida (Tabela 4).

Tabela 1 - Porcentagem de controle de Myriophyllum aquaticum aos sete dias após aplicação dos tratamentos químicos, sob diferentes intervalos sem chuva. Botucatu-SP, 2011

\begin{tabular}{|c|c|c|c|c|c|c|c|c|c|}
\hline \multirow{2}{*}{ Herbicida } & \multirow{2}{*}{$\begin{array}{c}\text { Dose } \\
\left(\mathrm{g} \mathrm{e}^{\mathrm{a} .} \mathrm{ha}^{-1}\right)\end{array}$} & \multicolumn{8}{|c|}{ Período sem chuva (horas) } \\
\hline & & 0,25 & 0,5 & 1,0 & 2,0 & 4,0 & 8,0 & 12,0 & Sem chuva \\
\hline $2,4-\mathrm{D}$ & 670 & $61,50 \mathrm{Bb}$ & $81,25 \mathrm{a}$ & $82,00 \mathrm{a}$ & $84,25 \mathrm{a}$ & $84,25 \mathrm{a}$ & $84,75 \mathrm{a}$ & $85,00 \mathrm{a}$ & $86,00 \mathrm{a}$ \\
\hline 2,4-D & 1.340 & $80,50 \mathrm{~A}$ & 84,50 & 83,00 & 84,25 & 84,50 & 85,00 & 85,50 & 86,75 \\
\hline \multicolumn{2}{|c|}{$\mathrm{F}_{\text {dose }}(\mathrm{D})$} & \multicolumn{8}{|c|}{$8,228^{* *}$} \\
\hline \multicolumn{2}{|c|}{$\mathrm{F}_{\text {chuva }}(\mathrm{C})$} & \multicolumn{8}{|c|}{$10,045^{* *}$} \\
\hline \multicolumn{2}{|c|}{$\mathrm{F}(\mathrm{D}) \mathrm{x}(\mathrm{C})$} & \multicolumn{8}{|c|}{$4,444 * *$} \\
\hline \multicolumn{2}{|c|}{$\mathrm{CV}(\%)$} & \multicolumn{8}{|c|}{5,3} \\
\hline \multicolumn{2}{|c|}{ d.m.s. (D) } & \multicolumn{8}{|c|}{6,19} \\
\hline \multicolumn{2}{|c|}{ d.m.s. (C) } & \multicolumn{8}{|c|}{9,76} \\
\hline
\end{tabular}

Médias seguidas da mesma letra, maiúscula na coluna e minúscula na linha, não diferem estatisticamente entre si pelo teste de Tukey $(\mathrm{p}<0,05) .{ }^{* *}$ significativo a $1 \%$ de probabilidade. 
Tabela 2 - Porcentagem de controle de Myriophyllum aquaticum aos 14 dias após aplicação dos tratamentos químicos, sob diferentes intervalos sem chuva. Botucatu-SP, 2011

\begin{tabular}{|c|c|c|c|c|c|c|c|c|c|}
\hline \multirow{2}{*}{ Herbicida } & \multirow{2}{*}{$\begin{array}{c}\text { Dose } \\
\left(\mathrm{g} \mathrm{e} \mathrm{a} \cdot \mathrm{ha}^{-1}\right)\end{array}$} & \multicolumn{8}{|c|}{ Período sem chuva (horas) } \\
\hline & & 0,25 & 0,5 & 1,0 & 2,0 & 4,0 & 8,0 & 12,0 & Sem chuva \\
\hline 2,4-D & 670 & $81,00 \mathrm{Bb}$ & $98,00 \mathrm{a}$ & $100,00 \mathrm{a}$ & $100,00 \mathrm{a}$ & $100,00 \mathrm{a}$ & $100,00 \mathrm{a}$ & $100,00 \mathrm{a}$ & $100,00 \mathrm{a}$ \\
\hline $2,4-\mathrm{D}$ & 1.340 & $97,75 \mathrm{~A}$ & 99,50 & 100,00 & 100,00 & 100,00 & 100,00 & 100,00 & 100,00 \\
\hline \multicolumn{2}{|c|}{$\mathrm{F}_{\text {dose }}(\mathrm{D})$} & \multicolumn{8}{|c|}{$5,317^{*}$} \\
\hline \multicolumn{2}{|c|}{$\mathrm{F}_{\text {chuva }}(\mathrm{C})$} & \multicolumn{8}{|c|}{$7,066^{* *}$} \\
\hline \multicolumn{2}{|c|}{$F(D) \times(C)$} & \multicolumn{8}{|c|}{$4,400 * *$} \\
\hline \multicolumn{2}{|c|}{$\mathrm{CV}(\%)$} & \multicolumn{8}{|c|}{4,0} \\
\hline \multicolumn{2}{|c|}{ d.m.s. (D) } & \multicolumn{8}{|c|}{5,63} \\
\hline \multicolumn{2}{|c|}{ d.m.s. (C) } & \multicolumn{8}{|c|}{8,87} \\
\hline
\end{tabular}

Médias seguidas da mesma letra, maiúscula na coluna e minúscula na linha, não diferem estatisticamente entre si pelo teste de Tukey $(\mathrm{p}<0,05) .{ }^{*}$ significativo a $5 \%$ de probabilidade. ${ }^{* *}$ significativo a $1 \%$ de probabilidade.

Tabela 3 - Porcentagem de controle de Myriophyllum aquaticum aos 21 dias após aplicação dos tratamentos químicos, sob diferentes intervalos sem chuva. Botucatu-SP, 2011

\begin{tabular}{|c|c|c|c|c|c|c|c|c|c|}
\hline \multirow{2}{*}{ Herbicida } & \multirow{2}{*}{$\begin{array}{c}\text { Dose } \\
\left(\text { g e.a. } \mathrm{ha}^{-1}\right)\end{array}$} & \multicolumn{8}{|c|}{ Período sem chuva (horas) } \\
\hline & & 0,25 & 0,5 & 1,0 & 2,0 & 4,0 & 8,0 & 12,0 & Sem chuva \\
\hline $2,4-\mathrm{D}$ & 670 & $95,25 \mathrm{~b}$ & $99,50 \mathrm{a}$ & $100,00 \mathrm{a}$ & $100,00 \mathrm{a}$ & $100,00 \mathrm{a}$ & $100,00 \mathrm{a}$ & $100,00 \mathrm{a}$ & $100,00 \mathrm{a}$ \\
\hline $2,4-\mathrm{D}$ & 1.340 & 98,75 & 100,00 & 100,00 & 100,00 & 100,00 & 100,00 & 100,00 & 100,00 \\
\hline \multicolumn{2}{|c|}{$F_{\text {dose }}(D)$} & \multicolumn{8}{|c|}{$1,182^{\mathrm{ns}}$} \\
\hline \multicolumn{2}{|c|}{$\mathrm{F}_{\text {chuva }}(\mathrm{C})$} & \multicolumn{8}{|c|}{$2,614^{*}$} \\
\hline \multicolumn{2}{|c|}{$F(D) \times(C)$} & \multicolumn{8}{|c|}{$0,886^{\mathrm{ns}}$} \\
\hline \multicolumn{2}{|c|}{$\mathrm{CV}(\%)$} & \multicolumn{8}{|c|}{1,9} \\
\hline \multicolumn{2}{|c|}{ d.m.s. (D) } & \multicolumn{8}{|c|}{2,62} \\
\hline \multicolumn{2}{|c|}{ d.m.s. (C) } & \multicolumn{8}{|c|}{4,12} \\
\hline
\end{tabular}

Médias seguidas da mesma letra minúscula na linha não diferem estatisticamente entre si pelo teste de Tukey (p<0,05). ${ }^{*}$ significativo a $5 \%$ de probabilidade. ${ }^{\text {ns }}$ não significativo.

Tabela 4 - Porcentagem de controle de Myriophyllum aquaticum aos 28 dias após aplicação dos tratamentos químicos, sob diferentes intervalos sem chuva. Botucatu-SP, 2011

\begin{tabular}{|c|c|c|c|c|c|c|c|c|c|}
\hline \multirow{2}{*}{ Herbicida } & \multirow{2}{*}{$\begin{array}{c}\text { Dose } \\
\left(\mathrm{g} \mathrm{e}^{-a .} \mathrm{ha}^{-1}\right)\end{array}$} & \multicolumn{8}{|c|}{ Período sem chuva (horas) } \\
\hline & & 0,25 & 0,5 & 1,0 & 2,0 & 4,0 & 8,0 & 12,0 & Sem chuva \\
\hline $2,4-\mathrm{D}$ & 670 & 97,00 & 100,00 & 100,00 & 100,00 & 100,00 & 100,00 & 100,00 & 100,00 \\
\hline 2,4-D & 1.340 & 99,50 & 100,00 & 100,00 & 100,00 & 100,00 & 100,00 & 100,00 & 100,00 \\
\hline \multicolumn{2}{|c|}{$\mathrm{F}_{\text {tratamento }}(\mathrm{T})$} & \multicolumn{8}{|c|}{$1,087^{\mathrm{ns}}$} \\
\hline \multicolumn{2}{|c|}{$\mathrm{F}_{\text {chuva }}(\mathrm{C})$} & \multicolumn{8}{|c|}{$2,130^{\mathrm{ns}}$} \\
\hline \multicolumn{2}{|c|}{$\mathrm{F}(\mathrm{T}) \mathrm{x}(\mathrm{C})$} & \multicolumn{8}{|c|}{$1,087^{\mathrm{ns}}$} \\
\hline \multicolumn{2}{|c|}{$\mathrm{CV}(\%)$} & \multicolumn{8}{|c|}{1,2} \\
\hline \multicolumn{2}{|c|}{ d.m.s. (D) } & \multicolumn{8}{|c|}{1,70} \\
\hline \multicolumn{2}{|c|}{ d.m.s. (C) } & \multicolumn{8}{|c|}{2,67} \\
\hline
\end{tabular}

Médias seguidas da mesma letra minúscula na linha não diferem estatisticamente entre si pelo teste de Tukey ( $p<0,05)$. ${ }^{\text {ns }}$ não significativo

Negrisoli et al. (2003) relataram que a aplicação do herbicida 2,4-D nas doses de 670 e $1.340 \mathrm{~g} \mathrm{ha}^{-1} \mathrm{em}$ plantas de $M$. aquaticum, sem posterior ocorrência de chuva, determinou 
controle total dos ramos, sendo que a partir dos 26 DAA não houve rebrotas, o que evidenciou um eficiente controle proporcionado por essas doses. Wersal \& Madsen (2010) observaram reduções maiores que $90 \%$ da biomassa dessa planta aquática com a aplicação do herbicida 2,4-D.

Os resultados obtidos confirmam a excelente eficácia do herbicida 2,4-D no controle de plantas de $M$. aquaticum mesmo com precipitações pluviais ocorridas logo após a aplicação do herbicida, como 15 minutos.

\section{LITERATURA CITADA}

ANDERSON, M. D.; ARNOLD, W. E. Weed control in sunflowers (Helianthus annuus) with desmediphan and phenmediphan. Weed Sci., v. 32, n. 3, p. 310-314, 1984.

DOMINGOS, V. D. et al. Alocação de biomassa e nutrientes em Myriophyllum aquaticum sob diferentes níveis de macronutrientes. Planta Daninha, v. 23, n. 2, p. 193-201, 2005.

GRAY, C. J. et al. Eurasian watermilfoil and parrotfeather control using carfentrazone-ethyl. J. Aquat. Plant Manag., v. 45, n. 1, p. $43-46,2007$.

HOFSTRA, D. E.; CHAMPION, P. D.; DUGDALE, T. M. Herbicide trials for the control of parrotsfeather. J. Aquat. Plant Manag., v. 44, n. 1, p. 13-18, 2006.

KISSMANN, K. G. Plantas infestantes e nocivas. 2.ed. São Bernardo do Campo: BASF, 1997. 852 p.

MacDONALD, E. G.; LANGELAND, A. K. Aquatic weed management alternatives for tropical areas. In: CONGRESSO DE LA ASOCIACION LATINOAMERICANA DE MALEZAS, 15., 2001, Maracaibo. Anais... Maracaibo: 2001. p. 44-47.

MARTINS, D. et al. Controle químico de Pistia stratiotes, Eichhornia crassipes e Salvinia molesta em caixas d'água. Planta Daninha, v. 20, p. 83-88, 2002. (Edição Especial)
MOREIRA, I.; MONTEIRO, A.; FERREIRA, T. Biology and control of parrotfeather (Myriophyllum aquaticum) in Portugal. Ecol. Environ. Conserv., v. 5, n. 3, p. 171-179, 1999.

NEGRISOLI, E. et al. Uso de diferentes herbicidas no controle de Myriophyllum aquaticum. Planta Daninha, v. 21, p. 89-92, 2003. (Edição Especial)

REYNOLDS, B. Invasive alien plant species of Virginia. Course requirements at Virginia Polytechnic Institute \& State University, 1999. Disponível em: <http:// www.hort.vt.edu>. Acesso em: 15 ago. 2011.

SOCIEDADE BRASILEIRA DA CIÊNCIA DAS PLANTAS DANINHAS - SBCPD. Procedimentos para instalação, avaliação e análise de experimentos com herbicidas. Londrina: 1995.42 p.

SOUZA, G. S. F. et al. Ação da chuva sobre a eficiência de glyphosate no controle de Eichhornia crassipes e Pistia stratiotes. Planta Daninha, v. 29, n. 1, p. 59-64, 2011.

WASHINGTON - Departament of Ecology Water Quality Program. Technical information about parrotfeather (Myriophyllum aquaticum). Disponível em: <http:// www. ecy.wa.gov/programs/wq/plants/weeds /aqua003.html $>$. Acesso em: 8 set. 2011

WERLANG, R. C. et al. Efeitos da chuva na eficiência de formulações e doses de glyphosate no controle de Brachiaria decumbens. Planta Daninha, v. 21, n. 1, p. 121-130, 2003.

WERSAL, R. M. et al. Comparison of daytime and night-time applications of diquat and carfentrazone-ethyl for control of parrotfeather and eurasian watermilfoil. J. Aquat. Plant Manag., v. 48, n. 1, p. 56-58, 2010.

WERSAL, R. M.; MADSEN, J. D. Comparison of subsurface and foliar herbicide applications for control of parrotfeather (Myriophyllum aquaticum). Invas. Plant Sci. Manag., v. 3, n. 3, p. 262-267, 2010.

WERSAL, R. M.; MADSEN, J. D. Comparison of imazapyr and imazamox for control of parrotfeather (Myriophyllum aquaticum (Vell.) Verdc.). J. Aquat. Plant Manag., v. 45, n. 2, p. 132-136, 2007. 